\title{
Design of IP tunneling for OSPF network to reduce advertising delay
}

\author{
Nattapong Kitsuwan ${ }^{\text {a) }}$ and Eiji Oki \\ Department of Communication Engineering and Informatics, \\ The University of Electro-Communications, Tokyo, Japan
}

a)kitsuwan@uec.ac.jp

\begin{abstract}
An IP tunneling design scheme to reduce advertising delay in open shortest path first (OSPF) networks, in which the link load is required for traffic maintenance, is proposed. A controller and nodes are consisted in a network. A node sends an OSPF traffic engineering (OSPF-TE) advertisement when either link load or topology is changed. The controller determines and adjusts the routes in the network to avoid traffic congestion. The controller may experience delay in receiving advertisements due to OSPF forwarding delay. Therefore, route adjustment may be delayed. In the proposed scheme, an IP tunneling technique is used instead of the OSPF mechanism.
\end{abstract}

Keywords: network management, IP networks, open shortest path first traffic engineer (OSPF-TE), IP tunneling

Classification: Network Management/Operation

\section{References}

[1] E. Oki and A. Iwaki, "Load-balanced IP routing scheme based on shortest paths in hose model," IEEE Trans. Commun., vol. 58, no. 7, pp. 2088-2096, Jul. 2010. DOI:10.1109/TCOMM.2010.07.090219

[2] D. Katz et al., "Traffic Engineering (TE) Extensions to OSPF Version 2," RFC 3630, Sep. 2003.

[3] H. M. Alnuweiri, L. Y. K. Wong, and T. Al-Khasib, "Performance of new link state advertisement mechanisms in routing protocols with traffic engineering extensions," IEEE Commun. Mag., vol. 42, no. 5, pp. 151-162, 2004. DOI:10.1109/ MCOM.2004.1299361

[4] Y. Koizumi, T. Miyamura, S. Arakawa, E. Oki, K. Shiomoto, and M. Murata, "Adaptive virtual network topology control based on attractor selection," IEEE/ OSA J. Light. Tech., vol. 28, no. 11, pp. 1720-1731, Jun. 2010. DOI:10.1109/JLT. 2010.2048412

[5] Y. Lee et al., "Path Computation Element Communication Protocol (PCEP) requirements and protocol extensions in support of global concurrent optimization," RFC 5557, Jul. 2009.

[6] C. Perkins, "IP encapsulation within IP," RFC 2003, Oct. 1996.

[7] "What is propagation delay? (Ethernet Physical Layer)", Ethernet FAQ, http:// stason.org/TULARC/networking/lans-ethernet/3-11-What-is-propagation-delayEthernet-Physical-Layer.html, Oct. 21. 2010. 


\section{Introduction}

Adopting the most appropriate routing scheme can increase the network resource utilization and network throughput of Internet Protocol (IP) networks. Realizing the goal of the optimum assignment of resources to traffic will allow additional traffic to be supported. It will also suppress network congestion and increases robustness against the traffic demand fluctuations, most of which are difficult to predict. One useful approach to enhance routing performance is to minimize the maximum link utilization rate, called the network congestion ratio, of all network links [1].

The OSPF traffic engineering (OSPF-TE) protocol [2] is employed in OSPF networks for the purpose of traffic maintenance [3]. In the OSPF-TE network, traffic engineering link state advertisements (TE-LSAs), which consist of the LSA plus link load [1], are used to transfer link information. TE-LSA will be called an advertisement hereafter. Each node floods the advertisements over the links connected to itself. Network topology and a table that keeps link loads are built based on the information in the advertisements. Advertisements are created and transmitted upon startup and when either the network topology or the link load is changed.

The OSPF-TE network consists of a network controller and nodes, where the network controller is one of the OSPF peers in the network. The network controller is used to optimize routing in the network. When the controller receives an advertisement, the network congestion ratio is determined using information of link load in the advertisement. If the network congestion ratio exceeds a specified value, the network controller determines the appropriate routes and establishes them; it releases the path associated with the congested link and sets up another path. Route computation is performed using the advertised traffic demands so as to minimize the network congestion ratio [4]. This is one application scenario, reoptimization of existing networks, in [5].

An advertisement travels from a node to the controller via the shortest path, which refers to network cost. This paper takes delay as the network cost. The route over which the advertisement first reaches the controller in the shortest time is the shortest path. The advertisement is forwarded from node to node until it reaches the controller.

IP tunneling is a technique to encapsulate one IP datagram within another IP datagram, so that a datagram destined for one IP address can be warped and redirected to another IP address. The link between the warped source and destination IP addresses is called a tunnel. An IP packet is encapsulated into another IP packet, called a capsule, at a tunnel ingress node [6]. The capsule is routed through the tunnel to the egress node of the tunnel. At the egress node, the IP packet is recovered by unpacking the capsule. In a tunnel, advertisements are forwarded from one node to the next node as the information in the routing table. The advertisement is received and forwarded immediately. Therefore, forwarding in the IP tunnel is faster than by OSPF mechanism.

This paper proposes an IP tunneling design scheme to reduce advertising delay in OSPF networks. IP tunnels are set between the controller and several nodes in the network. We design IP tunneling by selecting the set of nodes for the IP tunnels 


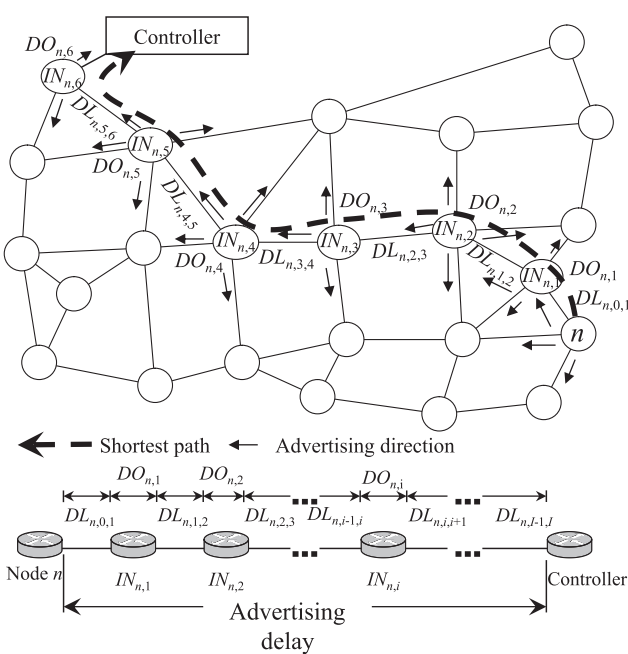

(a) Advertising delay from node $n$ to controller in conventional scheme.

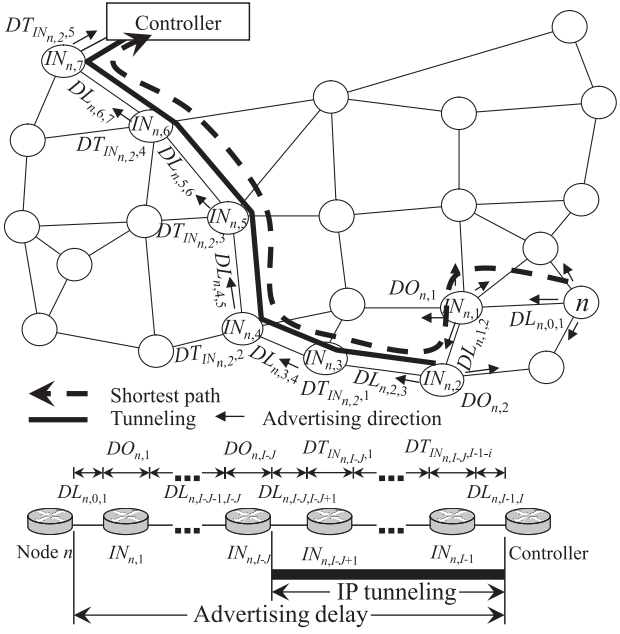

(b) Advertising delay from node $n$ to controller in IP tunneling scheme.

Fig. 1. Schematic of routing and advertising delay from node $n$ to controller in conventional and IP tunneling schemes.

(IP t-nodes) to minimize the advertising delay. Evaluation results show that the advertising delay can be decreased by $30 \%$ compared to the conventional scheme.

\section{Conventional scheme}

The OSPF forwarding mechanism is shown in Fig. 1(a). Node $n$ sends an advertisement to the controller on the shortest path via $I N_{n, 1}, \ldots, I N_{n, 6}$. At each intermediate node, the advertisement is copied and forwarded to the next node.

Let $I N_{n, i}$ be an intermediate node on the shortest path between node $n$ and the controller, where $i$ is the hop index from node $n$. $I$ is the number of hops on the path, so $I N_{n, I}$ is the controller. Let $D L_{n, i, i+1}$ be the propagation delay of the link between $I N_{n, i}$ and $I N_{n, i+1}$. Let $D O_{n, i}$ be OSPF forwarding delay at $I N_{n, i}$. The advertising delay from node $n$ to the controller, $T_{n}^{c o n}$, which is the delay between the output port of node $n$ and input port of the controller, shown in Fig. 1(a), is given by

$$
T_{n}^{c o n}=\sum_{i=0}^{I-1} D L_{n, i, i+1}+\sum_{i=1}^{I-1} D O_{n, i}
$$

Average advertising delay, $\overline{T_{n}^{c o n}}$, is the average value of $T_{n}^{c o n}$, which is $\overline{T_{n}^{c o n}}=$ $\frac{\sum_{n=1}^{N} T_{n}^{c o n}}{N}$, where $N$ is the number of nodes in the network.

\section{Proposed IP tunneling design scheme}

IP tunnels are set between the controller and nodes in the network. At each intermediate node in the tunnel, IP forwarding delay occurs instead of OSPF forwarding delay.

Parameters that impact the delay are the number of IP tunnels and the set of the tunnel entrance nodes (IP t-nodes). The number of IP tunnels that satisfies the requirement of delay is investigated. Advertising delay from each node to the controller is considered by a shortest path. Average advertising delay is an average value of all advertising delays between each node and the controller. The set of IP t- 
nodes that achieves the lowest average advertising delay is selected. The number of IP tunnels that satisfy the requirement of delay is decided.

Fig. 1(b) shows advertisement passing from node $n$ to the controller in the proposed scheme. Node $n$ floods an advertisement to every neighbor node. At $I N_{n, 1}$ and $I N_{n, 2}$, the advertisement is received, copied, and sent to the neighbor nodes. The advertisement from $I N_{n, 2}$ is then warped to the controller via $I N_{n, 3}, \cdots, I N_{n, 7}$. The advertising delay is the summation of link propagation delays, OSPF forwarding delays at $I N_{n, 1}$ and $I N_{n, 2}$, and IP forwarding delays from $I N_{n, 3}$ to $I N_{n, 7}$.

Advertising delay from node $n$ to the controller is shown in Fig. 1(b). A tunnel begins at $I N_{n, I-J}$, where $J$ is the number of hops from IP t-node, $I N_{n, I-J}$, to the controller. Let $D T_{I N_{n, I-J}, j}$ be the IP forwarding delay at $I N_{n, I-J+j}$, where $j$ is the number of hops from $I N_{n, I-J}$. Advertising delay from node $n$ to the controller, $T_{n}^{p r o}$, becomes

$$
T_{n}^{p r o}=\sum_{i=0}^{I-1} D L_{n, i, i+1}+\sum_{i=1}^{I-J} D O_{n, i}+\sum_{j=1}^{J-1} D T_{I N_{n, l-J}, j} .
$$

Average advertising delay, $\overline{T_{n}^{\text {pro }}}$, in the network is the average value of $T_{n}^{\text {pro }}$ which is given by $\overline{T_{n}^{p r o}}=\frac{\sum_{n=1}^{N} T_{n}^{p r o}}{N}$.

\section{Performance evaluation}

\subsection{Measuring the advertisement passing delay}

OSPF forwarding and IP forwarding delays, needed to evaluate the potential of the proposed scheme, were measured using the experimental setup shown in Fig. 2. $L S_{C}$, who receives the same packet twice, determines the time difference between the two packets. The time difference with and without tunneling are called IP forwarding delay and OSPF forwarding delay, respectively.

In the experiment, computers with AMD Phenom ${ }^{\mathrm{TM}}$ II X6 1090T Processor CPU and 8 GB of DDR3 memory on an M4A89GTD PRO/USB3 mother board are used as routers. Measured delays are $0.2 \mathrm{~ms}$ and $0.0075 \mathrm{~ms}$ per node, which will be referred to OSPF forwarding delay and IP forwarding delay, respectively.

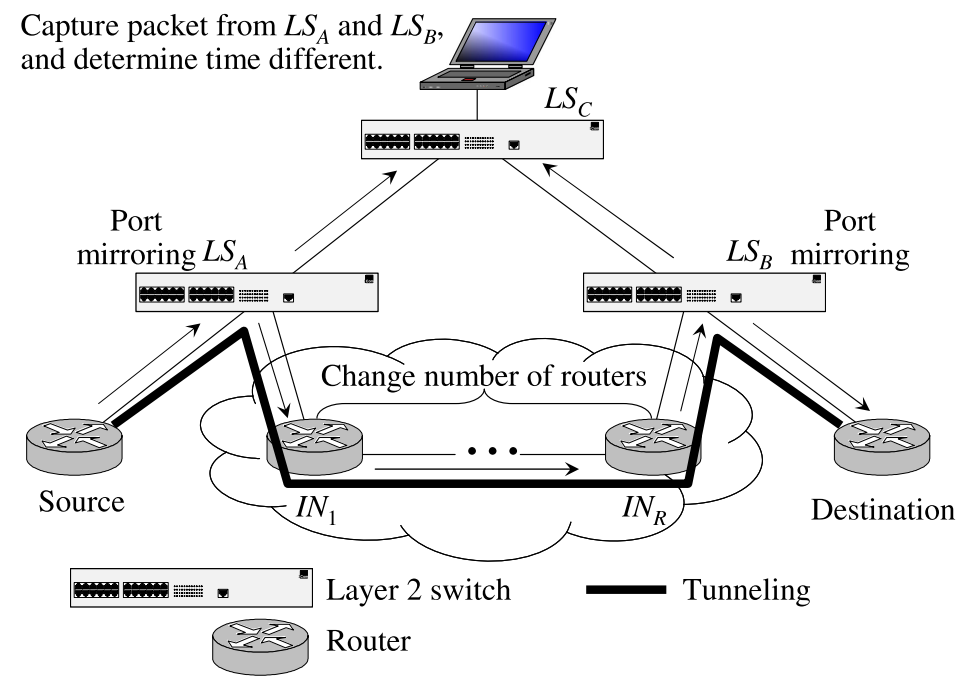

Fig. 2. Experimental setup for delay measurements. 


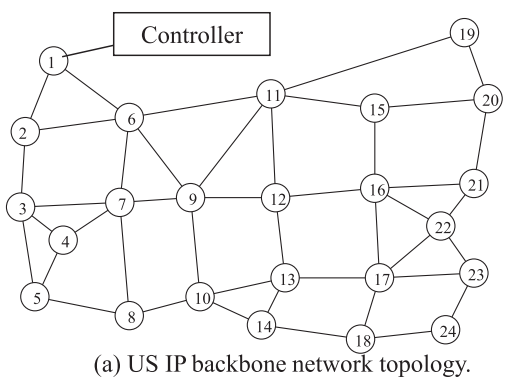

(a) US IP backbone network topology.

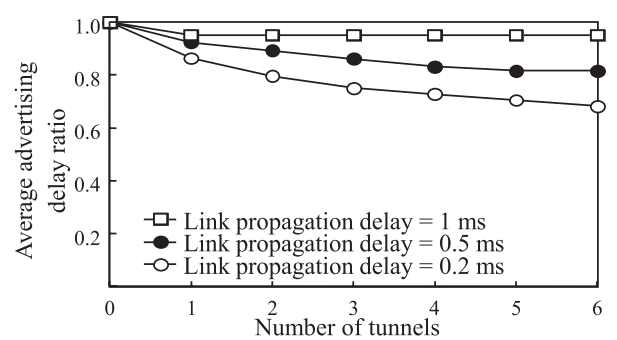

(b) Average advertising delay ratio vs. tunnel number in US IP backbone network topology.

Fig. 3. Average advertising delay ratio vs. tunnel number.

\subsection{Evaluation in large networks}

The performance of the proposed scheme is compared to the original OSPF forwarding mechanism in terms of average advertising delay. The simulation considers the US IP backbone network topology, which consists of 24 nodes and 43 cables, as in Fig. 3(a). The propagation speed is $231,000 \mathrm{~km} / \mathrm{sec}$ [7]. The number of tunnels is set in the range from zero to six. Zero tunnel indicates the conventional scheme. The optimum set of IP t-nodes when the number of IP tunnels is $1,2,3,4,5$, and 6 are $\{12\},\{10,11\},\{3,10,11\},\{3,10,11,16\}$, $\{7,10,11,16,24\}$, and $\{3,10,11,12,16,17\}$, respectively. Three cable lengths are investigated: $46.2 \mathrm{~km}, 115.5 \mathrm{~km}$, and $231 \mathrm{~km}$ (their propagation delays are $0.2 \mathrm{~ms}$, $0.5 \mathrm{~ms}$, and $1 \mathrm{~ms}$, respectively). We consider only advertising delay.

To compare the performance of both schemes, we normalize the average advertising delay of the proposed scheme against that of the OPSF forwarding mechanism, called average advertising delay ratio. Let $m$ be the set of IP t-nodes. $\min \left(\overline{T_{n, m}^{p r o}}\right)$ is the minimum average advertising delay of $m$. The average advertising delay ratio becomes $\frac{\min \left(\overline{T_{n, m}^{p r o}}\right)}{\overline{T_{n}^{c o n}}}$.

Fig. 3(b) shows the performance of the proposed scheme. Average advertising delay ratio decreases as the number of tunnels is increased. Small link propagation delays are more effective than large ones since the advertising delay depends on two types of delays, link propagation delay and forwarding delay. Minimizing the propagation delay emphasizes the superiority of the IP forwarding delay. Assuming that the link propagation delay is $0.2 \mathrm{~ms}$, average advertising delay is decreased by $30 \%$ of when the number of tunnels is six. With link propagation delays of 1.0 and $0.5 \mathrm{~ms}$, the optimum numbers of tunnels are one and five, respectively.

\section{Conclusions}

An IP tunneling design scheme that reduces the advertising delay in OSPF networks was proposed. IP tunnels are set between the controller node and some other nodes, not all nodes. The advertisements are warped, instead of forwarding node by node as per the original OSPF forwarding mechanism, from the nodes to the controller, if the tunnels are included in the shortest paths. Therefore, the advertising delay of the proposed scheme is less than that of the OSPF forwarding mechanism. The number of IP tunnels and the set of nodes for IP tunneling that minimizes the advertising delay were investigated. We measured the IP and OSPF forwarding delays in an experiment. Moreover, we estimated the proposal's 
performance in an example network. The delay of the proposed scheme with six tunnels is approximately $30 \%$ lower than that of the original OSPF forwarding mechanism.

\section{Acknowledgment}

This work was supported in part by the Strategic Information and Communications R\&D Promotion Programme of the Ministry of Internal Affairs and Communications, Japan. 